\title{
Clinical Effect of Xuefu Zhuyu Tang Combined with Atorvastatin on Hypertension Complicated with Coronary Heart Disease
}

\author{
Kaihua Long ${ }^{1}$, Xiaojia $\mathrm{Hu}^{1}$, Chunliu Wang ${ }^{2}$, Ye $\mathrm{Li}^{2}$ \\ ${ }^{1}$ Xi'an Peihua University, Shaanxi, Xi'an 710125, China; \\ ${ }^{2}$ Shaanxi Academy of Traditional Chinese Medicine, Shaanxi, Xi'an 710003, China.
}

Keywords: Xuefuzhuyu Decoction; atorvastatin; Hypertension complicated with coronary heart disease.

\begin{abstract}
Objective: To investigate the clinical efficacy of Rueful Zhou decoction combined with atorvastatin in the treatment of hypertension complicated by coronary heart disease. Method: Randomly January 2017 - December 201796 cases of coronary heart disease in patients with hypertension complicated hospital stay of the study, were randomly divided into control and observation groups were 48 cases. Aspirin, clopidogrel, and metoprolol were used for the basic treatment of the two groups of patients. At the same time, atorvastatin was given orally to both groups before bedtime, and the patients in the observation group were treated with Rueful Zhou Decoction. The course of treatment was: a month later, after the end of treatment compare the clinical effects of two groups of patients before and after treatment as well as blood pressure and blood flow change indicators as well as changes in cardiac function, while the treatment of the two groups of patients with total efficiency comparison. Results: The SBP and DBP after treatment in the two groups were significantly lower than before treatment $(\mathrm{P}<0.05)$. After treatment, the SBP and DBP levels in the control group were significantly higher than those in the observation group $(\mathrm{P}<0.05)$; after treatment, the two groups patients TC, TG, LDL-C were significantly lower than before treatment, HDL-C levels were significantly higher than before $(\mathrm{P}<0.05)$ treatment; treatment group observed after the TC, TG, LDL-C levels were significantly lower, The level of HDL-C in the control group was significantly lower than that in the observation group $(\mathrm{P}<0.05)$, and the difference was statistically significant. Conclusion: Rueful Zhou decoction combined with atorvastatin in the treatment of hypertension complicated by coronary heart disease is not only conducive to lower blood pressure, but also can effectively improve blood flow, has a good clinical effect, therefore, worthy of clinical application.
\end{abstract}

\section{Introduction}

Hypertension is a clinically common chronic disease that can easily result in coronary artery disease. It plays a very important role in the development of coronary heart disease. When the blood pressure continues rising, the resulting hemodynamic changes can activate platelets in the blood and cause atherosclerotic lesions, which in turn lead to myocardial hypoxia or necrosis, resulting in coronary heart disease [1]. At present, patients with hypertension have not recognized the risk factors that may generate coronary heart disease, and at the same time, the blood pressure control rate of most patients with coronary heart disease complicated with hypertension is less than $70 \%$. Therefore, it is needed to strengthen the management of risk factors in patients with hypertension complicated with coronary heart disease (hereinafter referred to as HCWCHD) in order to avoid the second attack [2]. However, as people's lifestyles are constantly changing, the number of patients with HCWCHD is also increasing. Clinically, attention has been given to constantly explore new ways to solve this problem. Studies have shown that Xuefu Zhuyu Decoction combined with atorvastatin has a very good therapeutic effect on HCWCHD [3]. 96 HCWCHD patients in our hospital were randomly selected as the study subjects, and 48 of them received Xuefu Zhuyu Decoction on the base the oral medication of atorvastatin, achieving good therapeutic effects. The specific content is reported as follows. 


\section{Data and Methods}

\subsection{General Data}

96 HCWCHD patients treated in our hospital from January 2017 to December 2017 were selected randomly as the study objects. They were randomly divided into the control group and the observation group (48 cases in each group), and all the patients were in line with the diagnostic criteria of hypertension and coronary heart disease. The control group included 21 female patients and 27 male patients aged between 45 and 78. The average age was $58.3 \pm 1.3$ years old and the average disease duration was $4.3 \pm 1.1$ years. The observation group was composed of 23 female patients and 25 male patients aged between 48 and 81 , and the average age was $61.3 \pm 1.6$ years old. There was no significant difference in the general data of all subjects between the two groups ( $p>0.05)$, and the test had statistically comparable significance.

\subsection{Methods}

Patients were asked not to take other antihypertensive drugs and anti-coronary heart disease drugs before and during the treatment. Aspirin, clopidogrel and metoprolol were used for basic treatment in the two groups of patients. At the same time, the patients were given atorvastatin for oral medication at bedtime, once a day and $10 \mathrm{mg}$ at a time. On this basis, patients in the observation group were treated with Xuefu Zhuyu Decoction, one dose at a time and twice a day. The drug batch number: State Drug Approval Document 5100929Z0. The course of treatment was one month.

\subsection{Evaluation Standards}

The treatment effects of the two groups were divided into: obviously effective treatment, effective treatment and ineffective treatment. The total effective rate was calculated on the base of the rate of "obviously effective treatment" and the rate of "effective treatment", and the total effective rate = the rate of obviously effective treatment + the rate of effective treatment. The total effective rate is expressed as a percentage. The higher the percentage of the total effective rate, the better the treatment effect is. It was evaluated as obviously effective treatment if the diastolic pressure was maintained within the normal range after blood pressure dropped. It was rated as effective treatment if the diastolic blood pressure dropped by $10-20 \mathrm{mmHg}$, or if the decreased by less than $10 \mathrm{mmHg}$ but the falling blood pressure remained within the normal range. It was assessed to be ineffective treatment if the blood pressure did not drop or the decrease was small.

\subsection{Observation Targets}

Patients of the two groups were compared in the curative effect of hypertension, the curative effect of coronary heart disease, the change of SBP, DBP, TC, TG, LDL-C and HDL-C as well as the change of diastolic blood pressure and systolic blood pressure before and after the treatment.

\subsection{Statistical Analysis}

The statistical data involved in this experiment was all sorted by computer (statistical software SPSS22.0). In order to ensure the statistical accuracy, all numerical values were the average value of three treatments. The statistical operation adopted descriptive analysis and inferential analysis. In the statistics, measurement and enumeration data were expressed as $(\mathrm{x} \pm \mathrm{s})$ and percentage $(\%)$, while $\mathrm{t}$ and $\mathrm{x} 2$ tests were used for the comparison between the two groups. The result value was represented by $\mathrm{P}$, and $\mathrm{p}<0.05$ indicated that the results had significant statistical difference.

\section{Results}

\subsection{Comparison of Total Effective Rate between Patients of the Two Groups}

In the control group, there were 26 cases enjoyed obviously effective treatment, 19 effective and 3 ineffective, and the total effective rate was $93.75 \%$. In the observation group, 29 patients enjoyed obviously effective treatment, 18 effective and 1 ineffective, and the total effective rate was $97.92 \%$. The differences between the two groups were statistically significant $(\mathrm{P}<0.05)$, as shown in Table 1: 
Table 1. Comparison of the total effective rate between patients of the two groups

\begin{tabular}{ccccc}
\hline Group & $\begin{array}{c}\text { Rate of obviously } \\
\text { effective treatment }\end{array}$ & $\begin{array}{c}\text { Rate of effective } \\
\text { treatment }\end{array}$ & $\begin{array}{c}\text { Rate of ineffective } \\
\text { treatment }\end{array}$ & $\begin{array}{c}\text { Total } \\
\text { effective rate }\end{array}$ \\
\hline $\begin{array}{c}\text { Control group } \\
\text { Observation }\end{array}$ & $26(54.16 \%)$ & $19(39.58 \%)$ & $3(6.25 \%)$ & $45(93.75 \%)$ \\
group & $29(60.41 \%)$ & $18(37.5 \%)$ & $1(2.08 \%)$ & $47(97.92 \%)$ \\
x2 & 0.192 & & & 0.522 \\
P & $<0.05$ & & $<0.05$ \\
\hline
\end{tabular}

3.2 Comparison of Various Indicators in Two Groups of Patients before and After Treatment

In the observation group after treatment, the patients' SBP level was $1.58 \pm 0.26 \mathrm{~g} / \mathrm{L}$; the DBP level was $1.44 \pm 0.27 \mathrm{~g} / \mathrm{L}$; the HDL-C was $1.21 \pm 0.23 \mathrm{~g} / \mathrm{L}$; the TG was $1.31 \pm 0.34 \mathrm{~g} / \mathrm{L}$; the TC was $4.15 \pm 1.33 \mathrm{~g} / \mathrm{L}$ and the LDL-C was $2.78 \pm 0.35 \mathrm{~g} / \mathrm{L}$. In the control group, the patients' SBP level was $2.13 \pm 0.22 \mathrm{~g} / \mathrm{L}$; the DBP level was $2.45 \pm 0.29 \mathrm{~g} / \mathrm{L}$; the HDL-C was $2.24 \pm 0.31 \mathrm{~g} / \mathrm{L}$; the TG was $2.31 \pm 0.27 \mathrm{~g} / \mathrm{L}$; the TC was $5.11 \pm 1.41 \mathrm{~g} / \mathrm{L}$, and the LDL-C was $4.02 \pm 0.89 \mathrm{~g} / \mathrm{L}$. The differences between the two groups were statistically significant $(\mathrm{P}<0.05)$, as shown in Table 1:

Table 2. Comparison of various indicators in two groups of patients before and after treatment

\begin{tabular}{|c|c|c|c|c|c|c|}
\hline Group/n & SBP & HDL-C & TG & $\mathrm{TC}$ & LDL-C & DBP \\
\hline $\begin{array}{c}\text { Observation } \\
\text { group/48 }\end{array}$ & $1.58 \pm 0.26 \mathrm{~g} / \mathrm{L}$ & $1.21 \pm 0.23 \mathrm{~g} / \mathrm{L}$ & $1.31 \pm 0.34 \mathrm{~g} / \mathrm{L}$ & $4.15 \pm 1.33 \mathrm{~g} / \mathrm{L}$ & $2.78 \pm 0.35 \mathrm{~g} / \mathrm{L}$ & $\begin{array}{c}1.44 \pm \\
0.27 \mathrm{~g} / \mathrm{L}\end{array}$ \\
\hline $\begin{array}{l}\text { Control } \\
\text { group/48 }\end{array}$ & $2.13 \pm 0.22 \mathrm{~g} / \mathrm{L}$ & $2.24 \pm 0.31 \mathrm{~g} / \mathrm{L}$ & $2.31 \pm 0.27 \mathrm{~g} / \mathrm{L}$ & $5.11 \pm 1.41 \mathrm{~g} / \mathrm{L}$ & $4.02 \pm 0.89 \mathrm{~g} / \mathrm{L}$ & $\begin{array}{c}2.45 \pm \\
0.29 \mathrm{~g} / \mathrm{L}\end{array}$ \\
\hline $\mathrm{t}$ & 17.188 & 22.889 & 25.641 & 4.706 & 9.688 & 24.048 \\
\hline$P$ & $<0.05$ & $<0.05$ & $<0.05$ & $<0.05$ & $<0.05$ & $<0.05$ \\
\hline
\end{tabular}

\section{Discussion}

In these days, hypertension and coronary heart disease have become common clinical diseases, and patients with hypertension are often accompanied by other chronic diseases. Some studies have shown that the blood pressure level is closely related to coronary heart disease, specifically, the higher the blood pressure level, the higher the incidence of coronary heart disease [4]. At present, many patients suffer HCWCHD and the incidence rate increases year by year. Therefore, effective measures must be taken to prevent the occurrence of hypertension and coronary heart disease. Atorvastatin not only plays an important role in bringing the high blood pressure down, but also can effectively prevent the occurrence of atherosclerosis and high cholesterol. In addition, it also plays a very important role in improving the remodeling of the ventricle, regulating immunity and preventing oxidation, and thus, atorvastatin is often used clinically as a basic drug for combination therapy [5]. Xuefu Zhuyu Decoction is a traditional Chinese medicine agent. It is a blood-regulating agent and has the effect of promoting blood circulation, activating blood, dissolving stasis, promoting qi circulation and relieving pain. In clinical practice, it is often used to treat coronary heart disease angina, rheumatic heart disease and chest pain caused by chest contusion and costicartilage inflammation. At the same time, it is also used for the treatment of cerebral thrombosis, hypertension, hyperlipidemia, thromboangiitis obliterans, neurosis as well as headache, dizziness and some other symptoms caused by brain concussion sequelae [6].

In this study, patients of both groups used aspirin, clopidogrel and metoprolol for basic treatment, and at the same time, all of them received oral medication of atorvastatin at bedtime. The patients in the observation group were treated with Xuefu Zhuyu Decoction for one month. The results showed that the total effective rate of treatment in the observation group was significantly higher than that in the control group, and the SBP and DBP after treatment in both groups were significantly lower than before treatment $(\mathrm{P}<0.05)$. After treatment, the SBP and DBP levels in the control group were significantly higher than that in the observation group $(\mathrm{P}<0.05)$. The levels of TC, TG and LDL-C in the two groups were significantly lower than before treatment, and the HDL-C levels were 
significantly higher than before treatment $(\mathrm{P}<0.05)$. The TC, TG and LDL-C levels in the observation group after treatment were significantly lower than that in the control group. The HDL-C levels in the control group were significantly lower than that in the observation group $(\mathrm{P}<0.05)$. The differences were statistically significant. The results obtained in this study are in accordance with the relevant literature reports. This shows that Xuefu Zhuyu Decoction combined with atorvastatin achieved a good therapeutic effect in the treatment of HCWCHD [7].

Atorvastatin plays a very important role in lowering plasma cholesterol and lipoprotein levels, which can not only reduce the production of low-density lipoproteins, but also help increase the number of LDL receptors on the surface of liver cells. In addition, it has significant curative effects on cardiovascular patients with coronary heart disease, ischemic stroke and diabetes as well as hypertension and other cardiovascular diseases, with high safety. As a traditional Chinese medical preparation, Xuefu Zhuyu Decoction is a blood agent having the effect of promoting blood circulation and relieving qi and relieving pain and is commonly used to treat hypertension caused by adverse blood gas in the clinic [8].

In summary, the application of Xuefu Zhuyu Decoction combined with atorvastatin on HCWCHD can effectively control blood pressure and reduce the incidence of coronary heart disease. Therefore, it should be promoted in clinical applications.

\section{References}

[1]. Mei Guangyan, Li Jibe. Effect of Amlodipine Atorvastatin Calcium on the Blood Pressure and Blood Lipid Level in Patients with Hypertension Complicated with Coronary Heart Disease [J]. Journal of Clinical Medicine in Practice, 2016, 20(3):131-132.

[2]. Gaol Airboat, Ma Young, Wang Weiping. Observation of Curative Effect of Atorvastatin Calcium Tablets in the Treatment of Hypertension Complicated with Coronary Heart Disease in 50 Patients [J]. Shaanxi Medical Journal, 2016, 45(9): 1244-1246.

[3]. Liu Buying, Zhang Bin, Wang Muhua, et al. Efficacy of Amlodipine Atorvastatin Calcium Tablets in the Treatment of Hypertension Complicated with Coronary Heart Disease [J]. Chinese Journal of Medical Guide, 2016, 18(9): 927-928.

[4]. Yang Shumen, Zhang Sheng, Fu Jing, et al. Amlodipine and Atorvastatin Calcium Tablets on Hypertension Complicated with Coronary Heart Disease Clinical Analysis [J]. Progress in Modern Biomedicine, 2013, 13(1): 116-118.

[5]. Sheng Weidman, Zhou You Hua, Zhao Hanau, et al. Clinical effect of Xuezhikang combined with atorvastatin in the treatment of elderly patients with hypertension complicated with coronary heart disease [J]. Hainan Medical Journal, 2013, 24(22): 3306-3307.

[6]. Cheng Meowed. Observation of Curative Effect of Xuefu Zhuyu Decoction on the Acute Cerebral Infarction $[\mathrm{J}]$. Chinese Journal of Integrative Medicine on Cardio/Cerebrovascular Disease, 2017, 15(10):1274-1276.

[7]. Zhou Yong, Men Jiangsu, Zhang Heng, et al. Influence of Xuefu Zhuyu decoction combined with atorvastatin for the vascular function state and ventricular remodeling state of elderly pat. 